\title{
Subjective memory deterioration in elderly people is associated with future dementia
}

Wang L, van Belle G, Crane PK, et al. Subjective memory deterioration and future dementia in people aged 65 and older. J Am Geriatr Soc 2004;52:2045-51.

Does subjective memory deterioration predict future dementia in elderly people with normal, non-demented, cognitive function?

METHODS

Design: Prospective cohort study.

$\sum$

Follow up period: 5.2 years (mean follow up).

Setting: One health maintenance organisation, Seattle, USA 1994-2002.

을 People: 1883 community dwelling elderly adults (65 years and over) without dementia and with normal cognitive function (score $>90$ out of 100 on the Cognitive Ability Screening Instrument, CASI). Exclusions: already enrolled in a clinical study or lacking baseline data on subjective memory change. Refusal to participate was more common in people $>84$ years old, AfricanAmericans and people from minority ethnic groups.

1

Risk factors: Participants were screened every 2 years and people with a CASI score $<86$ underwent clinical examination for dementia. People diagnosed with DSM-IV dementia were reassessed yearly to confirm the diagnosis. The Subjective Memory Rating Scale (SMRS, score range 5-25, higher score means greater deterioration) was used to measure subjective memory change. It assesses whether participants' ability to remember faces, friends, names and appointments and judge time had changed in the past 10-20 years (at baseline) or since their last assessment (at follow up). Subjective memory change was defined as an SMRS score of 20 and over. Cox regression was carried out to calculate hazard ratios and $95 \%$ confidence intervals.

Outcomes: Dementia (National Institute of Neurological and Communicative Disease and Stroke/Alzheimer's Disease and Related Disorder classification for Alzheimer's disease; DSM-IV for other dementia types)

\section{MAIN RESULTS}

At 5 years, 126 participants $(6.7 \%)$ developed dementia. People with subjective memory deterioration (SMD) at baseline were more likely to develop dementia than those without SMD at baseline (absolute risk of dementia: $15 \%$ in people with SMD baseline $v 6 \%$ in people without SMD, significance not stated). People reporting subjective memory deterioration at age 70 or 75 were significantly more likely to develop dementia than those not reporting subjective memory deterioration at these ages (age 70: HR 6, 95\% CI 2.1 to 18; age 75: HR 3, $95 \%$ CI 1.6 to 6.2 ). However, there was no significant difference in the risk of dementia between people with and without subjective memory deterioration at age 80 (HR 2, 95\% CI 0.9 to 3.1 ).

\section{CONCLUSIONS}

Subjective memory deterioration among younger elderly people (age 70 or 75 ) with normal cognitive function is associated with an increased risk of developing dementia. Measuring subjective memory may help identify people at high risk of developing dementia.

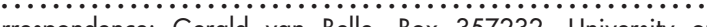
For correspondence: Gerald van Belle, Box 357232, University of Washington, Seattle WA 98195, USA; vanbelle@u.washington.edu

Sources of funding: National Institute on Aging, National Institute of Health, USA.

\section{NOTES}

Authors note that the SMRS has limited clinical use in its present form and that better instruments for measuring subjective memory are needed.

\section{Commentary}

ubjective memory complaints in dementia assessment and diagnosis represent an important area of inquiry, especially in the light of Petersen's inclusion of memory complaints in the definition of mild cognitive impairment (MCl). ${ }^{1-4}$ Subjective memory complaints have been shown to be more highly associated with depression in late life than with objective memory impairments. ${ }^{5}$ However, most of these studies have been cross-sectional and few, if any, have specifically addressed whether subjective memory deterioration predicts future dementia. Since $\mathrm{MCl}$ represents a risk factor for conversion to Alzheimer's disease (AD) ${ }^{1}$ the issue of whether this controversial criterion of subjective memory decline relates to future dementia is a critical empirical question.

Wang et al present a community based longitudinal study of over 1800 older adults investigating the temporal relationship between subjective memory decline and future dementia. In short, they found that subjective memory deterioration significantly predicted the development of dementia. They also provided additional analyses demonstrating consistencies with previous findings (e.g. cross-sectional associations between subjective memory decline and depression) as well as important modifying relationships from other variables. For example, they showed that advanced age diminished the strength of prediction of future dementia. They conclude by suggesting that subjective memory decline provides additional information about future dementia at a time when objective cognitive impairment is not observed (that is, with screening instruments).

The clinical implications of this study, if confirmed, are clear: assessment of subjective memory decline in older adults may be an important additional component of both screening and comprehensive cognitive assessments. Moreover, there appears to be decreasing utility of this information with increasing age, perhaps due to increasing executive or self-monitoring deficits with advancing age or the possibility that memory problems are more ubiquitous among the very old $1 \geqslant 80$ years). Thus, distinguishing whether subjective memory decline is an early indicator of dementia or a concomitant aspect of normal ageing is more difficult in the very old. Regardless, the findings of Wang et al provide important new information on the prediction of future dementia from subjective estimations of memory decline.

Mark W Bondi, PhD, ABPP/CN Staff Neuropsychologist, VA San Diego Healthcare System, Associate Professor of Psychiatry, UC San Diego School of Medicine, San Diego, CA, USA

1 Petersen RC, Smith GE, Waring SC, et al. Mild cognitive impairment: clinical characterization and outcome. Arch Neurol 1999;56:303-8.

2 Petersen RC, Stevens JC, Ganguli M, et al. Practice parameter: Early detection of dementia: mild cognitive impairment (an evidence-based review). Neurology 2001;56:1133-42.

3 Petersen RC. Mild cognitive impairment as a diagnostic entity. J Intern Med 2004; 256:183-94.

4 Winblad B, Palmer K, Kivipelto M, et al. Mild cognitive impairmentbeyond controversies, towards a consensus: report of the International Working Group on Mild Cognitive Impairment. J Intern Med 2004;256:240-6.

5 Kaszniak AW, DiTraglia Christenson GD. Differential diagnosis of dementia and depression. In: M Storand, GR VandenBos (eds). Neuropsychological Assessment of Dementia and Depression in Older Adults: A Clinician's Guide. Washington DC: American Psychological Association, 1994:81118 